A. Yoshimoto

Nagoya Math. J.

Vol. 98 (1985), 67-76

\title{
ON A GENERALIZATION OF HAMBURGER'S THEOREM
}

\author{
AKINORI YOSHIMOTO
}

\section{Introduction}

The relationship between Poisson's summation formula and Hamburger's theorem [2] which is a characterization of Riemann's zetafunction by the functional equation was already mentioned in Ehrenpreis-Kawai [1]. There Poisson's summation formula was obtained by the functional equation of Riemann's zetafunction. This procedure is another proof of Hamburger's theorem. Being interpreted in this way, Hamburger's theorem admits various interesting generalizations, one of which is to derive, from the functional equations of the zetafunctions with Grössencharacters of the Gaussian field, Poisson's summation formula corresponding to its ring of integers [1]. The main purpose of the present paper is to give a generalization of Hamburger's theorem to some zetafunctions with Grössencharacters in algebraic number fields. More precisely, we first define the zetafunctions with Grössencharacters corresponding to a lattice in a vector space, and show that Poisson's summation formula yields the functional equations of them. Next, we derive Poisson's summation formula corresponding to the lattice from the functional equations.

\section{§1. Notations and formulation of the theorem}

We denote by $\boldsymbol{R}$ and $\boldsymbol{C}$ the field of real numbers and the field of complex numbers respectively. Let $F$ be an algebraic number field of degree $n$ with signature $\left[r_{1}, r_{2}\right]$. We can naturally embed $F$ into $R^{r_{1}} \times$ $\boldsymbol{C}^{r_{2}}$. Put $V=\boldsymbol{R}^{r_{1}} \times \boldsymbol{C}^{r_{2}}$. Then $V$ may be regarded as a commutative ring. For $x=\left(x^{(1)}, \cdots, x^{\left(r_{1}+r_{2}\right)}\right) \in V$, we put $\operatorname{tr} x=\sum_{p=1}^{r_{1}+r_{2}} \operatorname{tr}_{R} x^{(p)}, N x=$ $\prod_{p=1}^{r_{1}+r_{2}}\left|N_{R} x^{(p)}\right|, e(x)=\exp (2 \pi \sqrt{-1} \operatorname{tr} x)$. We define a lattice in $V$ as a subgroup of $V$ having a basis $\left\{\alpha_{1}, \cdots, \alpha_{n}\right\}$ independent over $R$. Let $L$ be a lattice in $V$. Then its dual can be defined as the set $L^{*}$ consisting of all $x \in V$ such that $e(x y)=1$ for all $y \in L$. We can show easily that $L^{*}$

Received January 11, 1984. 
is a lattice in $V$. Let $O_{F}$ be the ring of integers of $F$ and $E_{F}$ be the group of units of $F$. Throughout this paper, we consider only $L$ satisfying $O_{F} L \subset L$.

Next, if $D$ is a real number satisfying $D \geqq 1$, we define the functions $A_{(a, b)}^{L}(s), B_{(a, b)}^{L^{*}}(s)$ as follows:

$$
\begin{aligned}
& A_{(a, b)}^{L}(s)=\sum_{(m)} \frac{c_{m} \lambda_{a}(m) \bar{\mu}_{b}(m)}{N m^{s}}, \\
& B_{(a, b)}^{L *}(s)=\sum_{(\nu)} \frac{d_{\nu} \lambda_{a}(\nu) \bar{\mu}_{b}(\nu)}{N \nu^{s}},
\end{aligned}
$$

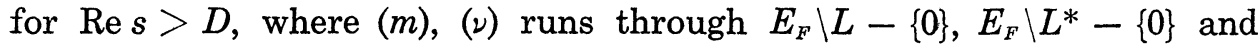
$c_{m}, d_{\nu}$ are complex numbers. Moreover we define

$$
\begin{aligned}
& \lambda_{a}(x)=\prod_{p=r_{1}+1}^{r_{1}+r_{2}}\left(\frac{x^{(p)}}{\left|x^{(p)}\right|}\right)^{a_{p}}, \\
& \mu_{b}(x)=\prod_{p=1}^{r_{1}+r_{2}}\left|x^{(p)}\right|^{\sqrt{-1} b_{p}},
\end{aligned}
$$

where $a=\left(a_{r_{1}+1}, \cdots, a_{r_{1}+r_{2}}\right), b=\left(b_{1}, \cdots, b_{r_{1}+r_{2}}\right)$ and $a_{p}\left(p=r_{1}+1, \cdots, r_{1}\right.$ $\left.+r_{2}\right)$ are integers and $b_{p}$ are real numbers such that $\sum_{p=1}^{r_{1}+r_{2}} b_{p}=0 . \vec{\mu}_{b}$ is complex conjugate to $\mu_{b}$, and for $\eta \in E_{F}$, we assume that $c_{\eta m}=c_{m}, d_{\eta \nu}=$ $d_{\nu}, \lambda_{a}(\eta)=1, \mu_{b}(\eta)=1$.

We further assume that $A_{(a, b)}^{L}(s)$ and $B_{(a, b)}^{L^{*}}(s)$ both absolutely converge in the domain $\{s \in C \mid \operatorname{Re} s>1\}$, and can be analytically continued to the whole plane as meromorphic functions having at most simple poles at $s=1$ if $(a, b)=(0,0)$ and can be analytically continued to the whole plane as entire functions if $(a, b) \neq(0,0)$. Moreover we assume that $(s-1) A_{(a, b)}^{L}(s)$ and $(s-1) B_{(a, b)}^{L^{*}}(s)$ are entire functions of finite order.

Let $\Phi(x)=\Phi\left(x^{(1)}, \cdots, x^{\left(r_{1}+r_{2}\right)}\right)$ be in $\mathscr{S}_{V}$ which is the Schwartz space over $V$ when we regard $V$ as $R^{n}$. Moreover we define

$$
\Phi^{*}(y)=\int_{V} \Phi(x) e(x y) d x,
$$

where

$$
\begin{gathered}
d x=d x^{(1)} \cdots d x^{\left(r_{1}\right)}\left|d x^{\left(r_{1}+1\right)} \wedge d \overline{x^{\left(r_{1}+1\right)}}\right| \cdots\left|d x^{\left(r_{1}+r_{2}\right)} \wedge d \overline{x^{\left(r_{1}+r_{2}\right)}}\right|, \\
x=\left(x^{(1)}, \cdots, x^{\left(r_{1}+r_{2}\right)}\right), \quad y=\left(y^{(1)}, \cdots, y^{\left(r_{1}+r_{2}\right)}\right) \in V .
\end{gathered}
$$

We put

$$
Z_{(a, b)}^{L}(s)=\sum_{(m)} \frac{\lambda_{a}(m) \bar{\mu}_{b}(m)}{N m^{s}},
$$


where the meaning of $(m)$ is the same as before.

We further put

$$
\begin{gathered}
M=\sum_{p=r_{1}+1}^{r_{1}+r_{2}}\left|a_{p}\right|, \quad N=\sum_{p=r_{1}+1}^{r_{1}+r_{2}} b_{p}, \quad A(L)=\left(\frac{2^{r_{1}} \cdot C(L)^{2}}{(2 \pi)^{n}}\right)^{1 / 2}, \\
c(L)=\left|\operatorname{det}\left(\begin{array}{cc}
\alpha_{1}^{(1)}, \cdots, \alpha_{1}^{\left(r_{1}+r_{2}\right)}, & \overline{\alpha_{1}^{\left(r_{1}+r_{2}\right)}} \\
\vdots & \vdots \\
\alpha_{n}^{(1)}, \cdots, \alpha_{n}^{\left(r_{1}+r_{2}\right)}, & \overline{\alpha_{n}^{\left(r_{1}+r_{2}\right)}}
\end{array}\right)\right| .
\end{gathered}
$$

Then our proposition and theorem are stated as follows:

Proposition. The functions $Z_{(a, b)}^{L}(s)$ satisfy

$$
\begin{aligned}
A(L)^{s} & \prod_{p=1}^{r_{1}}\left(\frac{s}{2}+\frac{\left|a_{p}\right|}{2}+\frac{\sqrt{-1} b_{p}}{2}\right) \prod_{p=1}^{r_{1}+r_{2}} \Gamma\left(s+\frac{\left|a_{p}\right|}{2}+\frac{\sqrt{-1} b_{p}}{2}\right) Z_{(a, b)}^{L}(s) \\
= & (-\sqrt{-1})^{M} \cdot 2^{\sqrt{-1} N} A\left(L^{*}\right)^{1-s} \prod_{p=1}^{r_{1}} \Gamma\left(\frac{1-s}{2}+\frac{\left|a_{p}\right|}{2}-\frac{\sqrt{-1} b_{p}}{2}\right) \\
& \times \prod_{p=r_{1}+1}^{r_{1}+r_{2}} \Gamma\left(1-s+\frac{\left|a_{p}\right|}{2}-\frac{\sqrt{-1} b_{p}}{2}\right) Z_{(-a,-b)}^{L^{*}}(1-s)
\end{aligned}
$$

for all $\lambda_{a}, \mu_{b}$.

TheOREM. Suppose that the functions $A_{(a, b)}^{L}(s)$ and $B_{(a, b)}^{L^{*}}(s)$ satisfy

$$
\begin{aligned}
A(L)^{s} & \prod_{p=1}^{r_{1}} \Gamma\left(\frac{s}{2}+\frac{\left|a_{p}\right|}{2}+\frac{\sqrt{-1} b_{p}}{2}\right) \prod_{p=r_{1}+1}^{r_{1}+r_{2}} \Gamma\left(s+\frac{\left|a_{p}\right|}{2}+\frac{\sqrt{-1} b_{p}}{2}\right) A_{(a, b)}^{L}(s) \\
= & (-\sqrt{-1})^{M} \cdot 2^{\sqrt{-1} N} A\left(L^{*}\right)^{1-s} \prod_{p=1}^{r_{1}}\left(\frac{1-s}{2}+\frac{\left|a_{p}\right|}{2}-\frac{\sqrt{-1} b_{p}}{2}\right) \\
& \times \prod_{p=r_{1}+1}^{r_{1}+r_{2}} \Gamma\left(1-s+\frac{\left|a_{p}\right|}{2}-\frac{\sqrt{-1} b_{p}}{2}\right) B_{(-a,-b)}^{L^{*}}(1-s)
\end{aligned}
$$

for all $\lambda_{a}, \mu_{b}$.

Then we have

$$
\sum_{m \in L} c_{m} \Phi^{*}(m)=c(L)^{-1} \sum_{\nu \in L^{*}} d_{\nu} \Phi(\nu) .
$$

Moreover the coefficients $c_{m}, d_{\nu}$ are all equal.

\section{§2. Proof of Proposition}

As is widely known, Poisson's summation formula is stated as follows:

$$
\sum_{m \in L} \Phi^{*}(m)=c(L)^{-1} \sum_{\nu \in L^{*}} \Phi(\nu)
$$


where $\Phi(x)$ is in $\mathscr{S}_{V}$. Put

$$
\Phi(x)=\exp \left(-\pi\left|x^{(1)}+u^{(1)}\right|^{2} t_{1}\right) \cdots \exp \left(-2 \pi\left|x^{\left(r_{1}+r_{2}\right)}+u^{\left(r_{1}+r_{2}\right)}\right|^{2} t_{r_{1}+r_{2}}\right)
$$

in (3), where $t_{1}, \cdots, t_{r_{1}+r_{2}}>0, u^{(1)}, \cdots, u^{\left(r_{1}\right)}$ are real variables and $u^{\left(r_{1}+1\right)}, \cdots$, $u^{\left(r_{1}+r_{2}\right)}$ are complex variables. Then we can readily show

$$
\begin{aligned}
& \sum_{m \in L} \exp \left(-\pi \sum_{p=1}^{n}\left|m^{(p)}+u^{(p)}\right|^{2} t_{p}\right) \\
& \quad=c(L)^{-1} \frac{1}{\sqrt{t_{1} \cdots t_{n}}} \sum_{\nu \in L^{*}} \exp \left(-\pi \sum_{p=1}^{n}\left|\nu^{(p)}\right|^{2} \frac{1}{t_{p}}+2 \pi \sqrt{-1} \sum_{p=1}^{n} \nu^{(p)} u^{(p)}\right),
\end{aligned}
$$

where

$$
\begin{array}{r}
u^{\left(r_{2}+p\right)}=\overline{u^{(p)}}, \quad t_{p+r_{2}}=t_{p}, \\
m^{\left(r_{2}+p\right)}=\overline{m^{(p)}}, \quad \nu^{\left(r_{2}+p\right)}=\overline{\nu^{(p)}} \\
\quad\left(p=r_{1}+1, \cdots, r_{1}+r_{2}\right) .
\end{array}
$$

Put $u^{(1)}=\cdots=u^{(n)}=0$ in (4). Then we obtain

$$
\begin{aligned}
& \sum_{m \in L} \exp \left(-\pi \sum_{p=1}^{n}\left|m^{(p)}\right|^{2} t_{p}\right) \\
& =c(L)^{-1} \sum_{\nu \in L^{*}} \exp \left(\sum_{p=1}^{n}\left|\nu^{(p)}\right|^{2} \frac{1}{t_{p}}\right) .
\end{aligned}
$$

We denote by $\boldsymbol{R}^{+}$the group of positive real numbers. We put $G=$ $\left(\boldsymbol{R}^{+}\right)^{r_{1}+r_{2}}$ and $\|t\|=\prod_{p=1}^{r_{1}} t_{p} \prod_{p=r_{1}+1}^{r_{1}+r_{2}} t_{p}^{2}$ for $t=\left(t_{1}, \cdots, t_{r_{1}+r_{2}}\right) \in G$. Moreover let $G^{0}$ be the subgroup consisting of all $y \in G$ such that $\|y\|=1$. Then we have a product $G=\boldsymbol{R}^{+} \times G^{0}$, where $\rho=\left(\rho^{1 / n}, \cdots, \rho^{1 / n}\right) \in G$ for $\rho \in \boldsymbol{R}^{+}$. Let $W$ be the image of the group of units $E_{F}$ in $G$, and $E$ be a fundamental domain for $W^{2}$ in $G^{0}$, where $W^{2}=\left\{x^{2} \mid x \in W\right\}$.

We denote the left hand side of (5) by $\theta(t, L)$. Then we have

$$
\theta(t, L)=c(L)^{-1} \frac{1}{\sqrt{\|t\|}} \theta\left(t^{-1}, L^{*}\right)
$$

On the other hand, we see

$$
\begin{aligned}
& \left(\frac{2^{r_{1}}}{(2 \pi)^{n}}\right)^{s / 2} \Gamma\left(\frac{s}{2}\right)^{r_{1}} \Gamma(s)^{r_{2}} Z_{(0,0)}^{L}(s) \\
& \quad=\int_{G} \sum_{(m)} \exp \left(-\pi \sum_{p=1}^{n}\left|m^{(p)}\right|^{2} t_{p}\right)\|t\|^{s / 2} \frac{d t}{t},
\end{aligned}
$$

where 


$$
\begin{aligned}
\frac{d t}{t} & =\frac{d t_{1}}{t_{1}} \cdots \frac{d t_{r_{1}+r_{2}}}{t_{r_{1}+r_{2}}}, \\
& =\frac{1}{W_{F}} \int_{0}^{\infty} \int_{E}(\theta(y c, L)-1) d^{*} c y^{s / 2} \frac{d y}{y},
\end{aligned}
$$

where $d^{*} c$ means the appropriate measure on $G^{0}, t=y c\left(y \in \boldsymbol{R}^{+}, c \in G^{0}\right)$, and $W_{F}$ is the number of roots of unity in $F$

$$
\begin{aligned}
= & \frac{1}{W_{F}} \int_{1}^{\infty} \int_{E}(\theta(y c, L)-1) d^{*} c y^{s / 2} \frac{d y}{y} \\
& \times \frac{1}{W_{F}} \int_{0}^{1} \int_{E}(\theta(y c, L)-1) d^{*} c y^{s / 2} \frac{d y}{y} .
\end{aligned}
$$

By using (6), we obtain

$$
\begin{aligned}
\int_{0}^{1} \int_{E} & (\theta(y c, L)-1) d^{*} c y^{s / 2} \frac{d y}{y} \\
= & -\frac{2 \mu^{*}(E)}{s}+\int_{0}^{1} \int_{E} \theta(y c, L) d^{*} c y^{s / 2} \frac{d y}{y} \\
= & -\frac{2 \mu^{*}(E)}{s}-\frac{2 \mu^{*}(E)}{(1-s)} c(L)^{-1} \\
& +\int_{1}^{\infty} \int_{E} c(L)^{-1}\left(\theta\left(y c, L^{*}\right)-1\right) d^{*} c y^{(1-s) / 2} \frac{d y}{y},
\end{aligned}
$$

where $\mu^{*}(E)$ denotes the integral of 1 over $E$ with respect to $d^{*} c$. Therefore we have

$$
\begin{aligned}
A(L)^{s} & \Gamma\left(\frac{s}{2}\right)^{r_{1}} \Gamma(s)^{r_{2}} Z_{(0,0)}^{L}(s) \\
& =A\left(L^{*}\right)^{1-s} \Gamma\left(\frac{1-s}{2}\right)^{r_{1}} \Gamma(1-s)^{r_{2}} Z_{(0,0)}^{L^{*}}(1-s),
\end{aligned}
$$

using $c(L) c\left(L^{*}\right)=1$.

We denote by $D_{a}$ the differential operator given as the product of all $\left(\partial / \partial u^{(p)}\right)^{a_{p}}\left(p=r_{1}+1, \cdots, r_{1}+r_{2}\right)$ for non-negative $a_{p}$ and all $\left(\partial / \partial u^{\left(p+r_{2}\right)}\right)^{-a_{p}}$ $\left(p=r_{1}+1, \cdots, r_{1}+r_{2}\right)$ for negative $a_{p}$. Assume $\left(a_{r_{1}+1}, \cdots, a_{r_{1}+r_{2}}\right) \neq$ $(0, \cdots, 0)$, and put $u^{(1)}=\cdots=u^{(n)}=0$ after operating $D_{a}$ on the both sides of (4), Then we obtain

$$
\begin{aligned}
& \sum_{m \in L} \lambda_{a}^{\prime}(m) \exp \left(-\pi \sum_{p=1}^{n}\left|m^{(p)}\right|^{2} t_{p}\right) \\
& \quad=c(L)^{-1} \frac{\prod_{p=r_{1}+1}^{r_{1}+r_{2}} t_{p}^{-\left|a_{p}\right|}}{\sqrt{t_{1} \cdots t_{n}}}(-\sqrt{-1})^{M} \sum_{\nu \in L^{*}} \lambda_{a}^{\prime}(\nu) \exp \left(-\pi \sum_{p=1}^{n}\left|\nu^{(p)}\right|^{2} \frac{1}{t_{p}}\right)
\end{aligned}
$$


with

$$
\lambda_{a}^{\prime}(m)=\lambda_{a}(m)\left|m^{\left(r_{1}+1\right)\left|a_{r_{1}+1}\right|} \cdots m^{\left(r_{1}+r_{2}\right)\left|a_{r_{1}+r_{2}}\right|}\right|, \quad \lambda_{a}^{\prime}(0)=0 .
$$

We denote the left hand side of $(7)$ by $\theta_{a}(t, L)$. Then we have

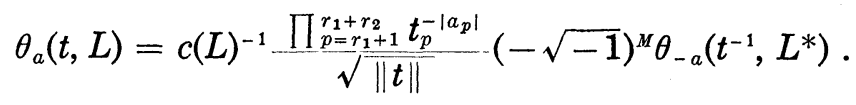

On the other hand, we see

$$
\begin{aligned}
& (2 \pi)^{-\stackrel{\substack{r_{1}+r_{2} \\
p=r_{1}+1}}{p} a_{p / 2} 2^{-\sqrt{-1}} \sum_{p=r_{1}+1}^{r_{1}+r_{2}} b_{p / 2}}\left(\frac{2^{r_{1}}}{(2 \pi)^{n}}\right)^{s / 2} \prod_{p=1}^{r_{1}} \Gamma\left(\frac{s}{2}+\frac{\left|a_{p}\right|}{2}+\frac{\sqrt{-1} b_{p}}{2}\right) \\
& \quad \times \prod_{p=r_{1}+1}^{r_{1}+r_{2}} \Gamma\left(s+\frac{\left|a_{p}\right|}{2}+\frac{\sqrt{-1} b_{p}}{2}\right) Z_{(a, b)}^{L}(s) \\
& =\frac{1}{W_{F}} \int_{G} \sum_{(m)} \lambda_{a}^{\prime}(m) \exp \left(-\pi \sum_{p=1}^{n}\left|m^{(p)}\right|^{2} t_{p}\right) \mu_{b / 2}(t) \prod_{p=r_{1}+1}^{r_{1}+r_{2}} t_{p}^{\left|a_{p}\right| / 2}\|t\|^{s / 2} \frac{d t}{t}
\end{aligned}
$$

with

$$
\begin{gathered}
\mu_{b / 2}(t)=\prod_{p=1}^{r_{1}+r_{2}} t_{p}^{\sqrt{-1} b_{p / 2}}, \\
=\frac{1}{W_{F}} \int_{0}^{\infty} \int_{E} \theta_{a}(y c, L) \mu_{b / 2}(y c) \prod_{p=r_{1}+1}^{r_{1}+r_{2}}\left(y c_{p}\right)^{\left|a_{p}\right| / 2} d^{*} c y^{s / 2} \frac{d y}{y}
\end{gathered}
$$

with

$$
\begin{aligned}
& c=\left(c_{1}, \cdots, c_{r_{1}+r_{2}}\right) \in G^{0}, \\
& =\frac{1}{W_{F}} \int_{1}^{\infty} \int_{E} \theta_{a}(y c, L) \mu_{b / 2}(y c) \prod_{p=r_{1}+1}^{r_{1}+r_{2}}\left(y c_{p}\right)^{\left|a_{p}\right| / 2} d^{*} c y^{s / 2} \frac{d y}{y} \\
& \quad+\frac{1}{W_{F}} \int_{0}^{1} \int_{E} \theta_{a}(y c, L) \mu_{b / 2}(y c) \prod_{p=r_{1}+1}^{r_{1}+r_{2}}\left(y c_{p}\right)^{|a p| / 2} d^{*} c y^{s / 2} \frac{d y}{y} .
\end{aligned}
$$

By using (8), we obtain

$$
\begin{aligned}
\int_{0}^{1} \int_{E} \theta_{a} & (y c, L) \mu_{b / 2}(y c) \prod_{p=r 1+1}^{r_{1}+r_{2}}\left(y c_{p}\right)^{\left|a_{p}\right| / 2} d^{*} c y^{s / 2} \frac{d y}{y} \\
= & \int_{1}^{\infty} \int_{E} \theta_{-a}\left(y c, L^{*}\right) \prod_{p=r_{1}+1}^{r_{1}+r_{2}}\left(y c_{p}^{-1}\right)^{-\left|a_{p}\right| / 2} \mu_{-b / 2}(y c) d^{*} c y^{(1-s) / 2} \frac{d y}{y} \\
& \cdot(-\sqrt{-1})^{M} \cdot c(L)^{-1} .
\end{aligned}
$$

Therefore we have

$$
A(L))^{s} \prod_{p=1}^{r_{1}} \Gamma\left(\frac{s}{2}+\frac{\left|a_{p}\right|}{2}+\frac{\sqrt{-1} b_{p}}{2}\right) \prod_{p=r_{1}+1}^{r_{1}+r_{2}} \Gamma\left(s+\frac{\left|a_{p}\right|}{2}+\frac{\sqrt{-1} b_{p}}{2}\right) Z_{(a, b)}^{L}(s)
$$




$$
\begin{aligned}
= & A\left(L^{*}\right)^{1-s} \prod_{p=1}^{r_{1}} \Gamma\left(\frac{1-s}{2}+\frac{\left|a_{p}\right|}{2}-\frac{\sqrt{-1} b_{p}}{2}\right) \prod_{p=r_{1}+1}^{r_{1}+r_{2}} \\
& \times \Gamma\left(1-s+\frac{\left|a_{p}\right|}{2}-\frac{\sqrt{-1} b_{p}}{2}\right) Z_{(-a,-b)}^{L^{*}}(1-s)(-\sqrt{-1})^{M} \cdot 2^{\sqrt{-1} N} .
\end{aligned}
$$

This completes the proof of the proposition.

\section{§3. Proof of Theorem}

Let $\Phi(x)$ be in $\mathscr{S}_{V}$. Put $\Phi_{e}(x)=\sum_{\varepsilon \in E_{F}} \Phi(\varepsilon x),\left|x^{(p)}\right|=\rho_{p}\left(p=1, \cdots, r_{1}\right)$, $x^{(p)}=\rho_{p} \exp \left(\sqrt{-1} \theta_{p}\right)\left(p=r_{1}+1, \cdots, r_{1}+r_{2}\right), \rho=\rho_{1} \cdots \rho_{r_{1}+r_{2}}^{2}$. Moreover let $P(\rho)$ be the set of $x=\left(x^{(1)}, \cdots, x^{\left(r_{1}+r_{2}\right)}\right) \in V$ with a common $\rho$. Then $\varepsilon \in E_{F}$ operates on $P(\rho)$ by $x \rightarrow \varepsilon x$ and $P(\rho)$ has a compact fundamental domain $E_{F} \backslash P(\rho)$. On the space $V$ we define a measure

$$
2^{r_{2}} \frac{d x^{(1)}}{\rho_{1}} \cdots \frac{d \rho_{r_{1}+r_{2}}}{\rho_{r_{1}+r_{2}}} d \theta_{r_{1}+1} \cdots d \theta_{r_{1}+r_{2}},
$$

and on $P(\rho)$ the induced measure $d P(\rho)$. Then $\Phi_{e}(x)$ has a multiplicative Fourier expansion

$$
\Phi_{e}(x)=\sum_{\lambda} \Psi_{\lambda}(\rho) \lambda(x),
$$

where $\lambda(x)=\lambda_{-a}(x) \overline{\mu_{-b}}(x)$, the sum extends over $\lambda$ satisfying the condition that $\lambda_{a}, \mu_{b}$ are well defined. Moreover

$$
\Psi_{\lambda}(\rho)=c \cdot \int_{E_{F} \backslash P(\rho)} \Phi_{e}(x) \bar{\lambda}(x) d P(\rho),
$$

where $c=\left(\int_{E_{F} \backslash P(\rho)} d P(\rho)\right)^{-1}$.

On the other hand, we see

$$
\Phi_{e}^{*}(x)=\sum_{\lambda} \hat{\Psi}_{\bar{\lambda}}(\rho) \bar{\lambda}(x),
$$

where $\Phi_{e}^{*}(x)=\sum_{\varepsilon \in E_{F}} \Phi^{*}(\varepsilon x)$. We further note

$$
\int_{V} \Psi_{\lambda}(\rho) \lambda(x) e(x y) d x=\Psi_{\lambda}\left(\rho^{\prime}\right) \bar{\lambda}(y),
$$

where $y=\left(y^{(1)}, \cdots, y^{\left(r_{1}+r_{2}\right)}\right),\left|y^{(p)}\right|=\rho_{p}^{\prime}\left(p=1, \cdots, r_{1}\right), y^{(p)}=\rho_{p}^{\prime} \exp \left(\sqrt{-1} \theta_{p}^{\prime}\right)$ $\left(p=r_{1}+1, \cdots, r_{1}+r_{2}\right), \rho^{\prime}=\rho_{1}^{\prime} \cdots \rho_{r_{1}+r_{2}}^{\prime 2}$. We assume that $r_{1} \neq 0$. Otherwise we can put $\rho=\rho_{1} \cdots \rho_{r_{2}}$ and prove Theorem similarly. We put $\rho_{1}^{\prime}$ $=t, \rho_{2}^{\prime}=\cdots=\rho_{r_{1}+r_{2}}^{\prime}=1, \theta_{r_{1}+1}^{\prime}=\cdots=\theta_{r_{1}+r_{2}}^{\prime}=0$ in (12), and take the integral of both sides with respect to $t$ from 0 to $\infty$ after multiplying 
them by $t^{s-1}$. Before proceeding further, we recall the following formulas:

$$
\begin{gathered}
J_{a}(z)=\frac{1}{2 \pi} \int_{\alpha}^{2 \pi+\alpha} \exp (\sqrt{-}-1(a \theta-z \sin \theta)) d \theta \\
\int_{0}^{\infty} x^{\mu} J_{a}(x y)(x y)^{1 / 2} d x=2^{\mu+1 / 2} y^{-\mu-1} \frac{\Gamma(\mu / 2+a / 2+3 / 4)}{\Gamma(-\mu / 2+a / 2+1 / 4)} \quad(y>0), \\
\int_{0}^{\infty} x^{s-1} \cos x d x=2^{s-1} \pi^{1 / 2} \frac{\Gamma(s / 2)}{\Gamma((1-s) / 2)},
\end{gathered}
$$

where $J_{a}(z)$ is the Bessel function. Thus by a direct computation using (13), (14) and (15), we have

$$
\begin{aligned}
M\left(\hat{\Psi}_{\bar{\lambda}}, s\right)= & \left(\frac{2^{r_{1}}}{(2 \pi)^{n}}\right)^{s-1 / 2} \sqrt{-1^{M}} 2^{-\sqrt{-1} N} \prod_{p=1}^{r_{1}} \frac{\Gamma\left(s / 2+\sqrt{-1} b_{p} / 2\right)}{\Gamma\left((1-s) / 2-\sqrt{-1} b_{p} / 2\right)} \\
& \times \prod_{p=r_{1}+1}^{r_{1}+r_{2}} \frac{\Gamma\left(s+\left|a_{p}\right| / 2+\sqrt{-1} b_{p} / 2\right)}{\Gamma\left(1-s+\left|a_{p}\right| / 2-\sqrt{-1} b_{p} / 2\right)} M\left(\Psi_{\lambda}, 1-s\right),
\end{aligned}
$$

where $M\left(\Psi_{\lambda}, s\right)=\int_{0}^{\infty} \Psi_{\lambda}(\rho) \rho^{s} d \rho / \rho$. Combining (16) with (2), we finally obtain

$$
M\left(\hat{\Psi}_{\bar{\lambda}}, s\right) A_{(a, b)}^{L}(s)=c(L)^{-1} M\left(\Psi_{\lambda}, 1-s\right) B_{(-a,-b)}^{L^{*}}(1-s) .
$$

Consider, on the other hand, the inverse Mellin transform of the left hand side of (17) along the line $\operatorname{Re} s=1 / 2$, and shift the integral to the line Re $s=\sigma_{1}\left(\sigma_{1}>D\right)$ using the Phramén-Lindelöf theorem. Then, provided that $(a, b) \neq(0,0)$, the result is

$$
\sum_{(m)} c_{m} \hat{\Phi}_{\bar{\lambda}}(m)
$$

where $\hat{\Phi}_{\bar{\lambda}}(m)=\hat{\Psi}_{\bar{\lambda}}(N m) \bar{\lambda}(m)$. Similarly, if we consider the inverse Mellin transform of the right hand side of (17) along the line $\operatorname{Re} s=1 / 2$, in turn shift the integral to the line $\operatorname{Re} s=1-\sigma_{1}\left(\sigma_{1}>D\right)$ provided that $(a, b) \neq$ $(0,0)$, then we have

$$
c(L)^{-1} \sum_{(\nu)} d_{\nu} \Phi_{\lambda}(\nu)
$$

where $\Phi_{\lambda}(\nu)=\Psi_{\lambda}(N \nu) \lambda(\nu)$. Therefore by (18) and (19), we obtain

$$
\sum_{(m)} c_{m} \hat{\Phi}_{\bar{\lambda}}(m)=c(L)^{-1} \sum_{(\nu)} d_{\nu} \Phi_{2}(\nu)
$$

Even if $(a, b)=(0,0)$, the same arguments lead to 


$$
\begin{aligned}
& \sum_{(m)} c_{m} \hat{\Phi}_{1}(m)-M\left(\hat{\Psi}_{1}, 1\right) A \\
& =c(L)^{-1} \sum_{(\nu)} d_{\nu} \Phi_{1}(\nu)-M\left(\Psi_{1}, 1\right) B \cdot c(L)^{-1},
\end{aligned}
$$

where we denote by $A, B$ the residue of $A_{(0,0)}^{L}(s), B_{(0,0)}^{L^{*}}(s)$ at $s=1$ respectively. Hence by (20), (21), and the definition of $\Phi_{e}(x)$, we obtain

$$
\begin{aligned}
\sum_{m \in L-\{0\}} c_{m} \Phi^{*}(m)+M\left(\Psi_{1}, 1\right) B \cdot c(L)^{-1} \\
=\sum_{\nu \in L^{*}-\{0\}} d_{\nu} \Phi(\nu)+M\left(\hat{\Psi}_{1}, 1\right) A .
\end{aligned}
$$

We can simplify the expression $M\left(\Psi_{1}, 1\right) B \cdot c(L)^{-1}$. It follows from (10) that

$$
\begin{aligned}
M\left(\Psi_{1}, 1\right) & =\int_{0}^{\infty} \Psi_{1}(\rho) \rho \frac{d \rho}{\rho} \\
& =\int_{0}^{\infty} c \cdot \int_{E_{F \backslash P(\rho)}} \Phi_{e}(x) d P(\rho) \rho \frac{d \rho}{\rho} \\
& =c \int_{0}^{\infty} \int_{P(\rho)} \Phi(x) d P(\rho) \frac{d \rho}{\rho} \\
& =c \cdot \Phi^{*}(0) .
\end{aligned}
$$

Similarly we have

$$
M\left(\hat{\Psi}_{\bar{\lambda}}, 1\right)=c \cdot \Phi(0) .
$$

Putting $c_{0}=c \cdot B \cdot c(L)^{-1}, d_{0}=c \cdot A \cdot c(L)$ and using (23) and (24), we obtain

$$
\sum_{m \in L} c_{m} \Phi^{*}(m)=c(L)^{-1} \sum_{\nu \in L^{*}} d_{\nu} \Phi(\nu) .
$$

Next we shall show that the coefficients $c_{m}, d_{\nu}$ in (25) are all equal. To do this we define $\psi_{0}(x)$ as a function on $V$ which vanishes except in a sufficiently small neighborhood of $x=0$ and satisfy the condition that $\psi_{0}(0)=1$. For $\ell \in L^{*}$, we put $\psi_{\ell}(x)=\psi_{0}(x-\ell)$, and put $\psi_{\ell}(x)=\psi_{0}(x-\ell)$ in (25). Then we have

$$
\begin{aligned}
c(L)^{-1} d_{\ell} & =c(L)^{-1} \sum_{\nu \in L^{*}} d_{\nu} \psi_{\ell}(\nu) \\
& =\sum_{m \in L} c_{m} \psi_{\ell}^{*}(m) .
\end{aligned}
$$

On the other hand, we see

$$
\begin{aligned}
\psi_{l}^{*}(m) & =\int_{V} \psi_{\ell}(x) e(x m) d x \\
& =\int_{V} \psi_{0}(x-\ell) e(x m) d x
\end{aligned}
$$




$$
\begin{aligned}
& =\int_{V} \psi_{0}(t) e(t m) d t e(\ell m), \\
& =\psi_{0}^{*}(m)
\end{aligned}
$$

with $t=x-\ell$, and note $e(l m)=1$ for all $m \in L$. Therefore we have $d_{\varepsilon}$ $=d_{0}$. Similarly, using $\Phi^{* *}(x)=\Phi(-x)$, we have $c_{m}=c_{0}$ for all $m \in L$. Thus we obtain

$$
c_{0} \sum_{m \in L} \Phi^{*}(m)=c(L)^{-1} d_{0} \sum_{\nu \in L^{*}} \Phi(\nu) .
$$

On the other hand, since $\sum_{m \in L} \Phi^{*}(m)=c(L)^{-1} \sum_{\nu \in L^{*}} \Phi(\nu)$ holds, we have $c_{0}=d_{0}$. This completes the proof of Theorem.

Remarks. 1. In the special case where $F=$ the field of rational numbers and $L=$ the ring of rational integers, our theorem reduces to Hamburger's theorem.

2. According to Hecke's theorem (Satz 176 in Hecke [5]), the different $\mathfrak{U}$ of a field $F$ can be expressed as $e \mathfrak{U}^{2}$, where $e \in F, \mathfrak{U}$ is an ideal of $F$. Hence if $F$ is a totally imaginary field and $L=(\sqrt{e} \mathfrak{U})^{-1}$, we obtain $L=L^{*}$.

\section{BIBLIOGRAPHY}

[1] L. Ehrenpreis and T. Kawai, Poisson's summation formula and Hamburger's theorem, Publ. Res. Inst. Math. Sci., 18 (1982), 413-426.

[2] H. Hamburger, Über die Riemannsche Funktionalgleichung der $\zeta$-Funktion, Math. Z., 10 (1921), 240-254.

[ 3 ] E. Hecke, Eine neue Art von Zetafunktionen und ihre Beziehungen zur Verteilung der Primzahlen, Math. Z., 1 (1918), 357-376.

[4] - Eine neue Art von Zetafunktionen und ihre Beziehungen zur Verteilung der Primzahlen, Math. Z., 6 (1920), 11-51.

[5] —- Vorlesungen über die Theorie der algebraischen Zahlen, Chelsea, (1970).

[6] T. Kubota, On an analogy to the Poisson summation formula for generalized Fourier transformation, J. reine angew. Math., 268 (1974), 180-189.

[ 7 ] S. Lang, Algebraic number theory, Addison-Wesley (1970).

Department of Mathematics

Faculty of Science

Kyushu University 33

Fukuoka 812

Japan

Current address:

Department of Mathematics

Faculty of Science

Nagoya University

Chikusa-ku, Nagoya 464

Japan 